\title{
Role of Flexible Bronchoscopy in Ventilator-Dependent Neonates
}

\author{
JaVeed IQbal Bhat, ${ }^{\mathbf{1}}$ Bashir ACharoo, ${ }^{1}$ Shihab Zahoor, ${ }^{1}{ }_{\text {QAZI Iqbal Ahmad }}{ }^{1}$ ANd Ambreen Ali Ahangar ${ }^{2}$ \\ From the Department of ${ }^{1}$ Pediatrics Sher-i-Kashmir Institute of Medical Sciences; and Department of ${ }^{2}$ Anesthesia, Government \\ Medical College; Srinagar, Jammu and Kashmir, India.
}

\author{
Correspondence to: Dr Javeed Iqbal \\ Bhat, Department of Pediatrics, \\ SKIMS, Soura, Jammu and Kashmir, \\ India.drjaveediqbal@gmail.com \\ Received: January 17, 2019; \\ Initial review: June 07, 2019; \\ Accepted: August 14, 2020.
}

Objective: To assess the usefulness and safety of flexible bronchoscopy in ventilated neonates with extubation failure. Method: This was a prospective observational study. Flexible bronchoscopy was done in eligible patients with failure of extubation form invasive ventilation. The main outcome measure was to find the presence of any anatomic or dynamic abnormalities of the airways of these patients and the organism profile of bronchoalveolar lavage (BAL) fluid. Results: Forty-eight babies (68.8\% preterm) were enrolled in the study. The most common finding on bronchoscopy was airway edema seen in $13(27 \%)$ patients. BAL culture was positive in $29(74 \%)$ patients. Overall treatment was modified in $35(73 \%)$ patients based on bronchoscopy findings/BAL culture. Majority of infants $(83.3 \%)$ tolerated the procedure very well. Conclusion: Flexible bronchoscopy provides useful information in the management of newborn babies with extubation failure.

Keywords: Bronchoalveolar lavage, Extubation failure, Management, Preterm.
$\mathrm{P}$ rolonged ventilation may lead to multiple adverse effects, including subglottic stenosis, tracheobronchomalacia, nosocomial infection, bronchopulmonary dysplasia (BPD) and neurocognitive impairment [1-3]. Up to $30 \%$ of mechanically ventilated infants require a prolonged period of invasive mechanical ventilation and experience repeated extubation failures. Kurachek, et al. [5], in their study on pediatric patients, reported that upper airway obstruction like subglottic stenosis, laryngo-malacia, tracheomalacia are the leading causes of extubation failure $[\mathrm{EF}]$. These observations demand prompt and precise diagnosis of these conditions. Similarly, ventilator-associated pneumonia (VAP) also increases the duration of mechanical ventilation.

Flexible bronchoscopy is a well-established tool for the evaluation of airway anomalies and infections in neonatal ICU, with excellent safety profile [6]. Moreover, therapeutic interventions with flexible bronchoscopy like mucus plug removal, can efficiently relieve airway problems and can decrease the duration of ventilation $[7,8]$. There is a paucity of literature regarding the role of flexible bronchoscopy in prolonged mechanical ventilation/failure of extubation in neonates. We studied the utility and safety of this modality in neonates on prolonged ventilation/extubation failure.

\section{METHODS}

We provide data on neonatal flexible bronchoscopy from
May, 2014 to April, 2018 at a tertiary-care public hospital of India. The study participants were neonates with a gestational age of more than 32 weeks and failure of extubation (defined by a need for re-intubation within 48 hours of extubation). Eligibility for and benefit of bronchoscopy were determined by the attending neonatologists. Written informed consent was taken from parents/legal guardians before undertaking the procedure. The study was approved by the institutional ethical committee.

Flexible bronchoscopy was done in the neonatal intensive care unit (NICU) or bronchoscopy suite, which is in close proximity to neonatal ICU. The bronchoscopy team comprised of a bronchoscopist, bronchoscopy technologist, neonatologist, pediatric resident doctor and a nurse. Stable ventilated neonates were extubated for the duration of the procedure in order to check for any upper airway anatomic and dynamic abnormality. Preoxygenation to ensure oxygen saturation remained above $90 \%$ was carried out. The majority of bronchoscopies were performed trans-nasally, the trans-oral route was used in four patients (cleft palate in two patients, choanal stenosis in one patient and epistaxis in one patient). In 10 patients, bronchoscopy was done via an endotracheal tube with a tube size of $3.5 \mathrm{~mm}$, because of high ventilator settings. Extubation was also attempted at some point in time in this sub-group. Upper airway could not be assessed in this subgroup. This subgroup consisted of six-term babies and four late preterm babies. The authors 
used Olympus BF-XP160F (Olympus Corporation, Japan) scope with an outer diameter of $2.8 \mathrm{~mm}$ and channel size of $1.2 \mathrm{~mm}$. Electrocardiogram and pulse were recorded continuously during the procedure and non-invasive blood pressure was monitored every 3-5 minutes. Supplemental oxygen was given via nasal cannula. Desaturation $\leq 90 \%$ was managed by an increase in oxygen flow rate and the use of an oxygen mask. $2 \%$ lidocaine gel was used locally to anesthetize nasal mucosa. $1 \mathrm{~mL}$ aliquots of $2 \%$ lidocaine in 1:1 dilution with normal saline were instilled by the 'spray-as-yougo' technique. Additional doses were given, if required, to minimize patient discomfort. Bronchoalveolar lavage (BAL) was performed with the use of normal saline warmed to body temperature with a volume of $3 \mathrm{~mL} / \mathrm{kg}$ administered in three divided doses. The bronchoscope was advanced until wedged in a desired subsegmental bronchus; this technique ensured the collection of a sample from the terminal airways with negligible contamination from the upper airways. It was sent for gram staining, lipid-laden macrophages, bacterial culture, and fungal culture. Bronchoscopy findings were noted if present. Tracheobronchial abnormalities recorded included subglottic stenosis, tracheomalacia (tracheomalacia or bronchomalacia was diagnosed when there was a $50 \%$ reduction in the luminal diameter during expiration), tracheal stenosis, complete tracheal rings, tracheoesophageal fistulas, vascular rings, bronchomalacia, hemangiomas, or mucus plugging. Therapeutic procedures carried out were also noted. BAL culture was done by using the BacT/Alert automatic culture system. Culture results including organism profile and culture sensitivity were recorded.

A standardized data extraction form was used to obtain the demographic and clinical data including patient age, sex, weight, co-morbidities, procedure indication, total midazolam dose, pulse rate, baseline and lowest blood pressure, oxygen saturation, adverse events if any during and/or within one hour of the procedure.

Statistical analysis: It was performed using SPSS 20.0. The normality of the data was checked by using the Shapiro- Wilk test. Categorical variables are presented as percentages and continuous data as mean (SD)/median (IQR).

\section{RESULTS}

During the study period, 998 newborn babies received mechanical ventilation for different indications; 48 of these $(68.8 \%$ preterm) underwent flexible bronchoscopy with or without BAL. The mean (SD) gestational age and birthweight of the study population was 36.4 (2.2) weeks and $2.5(0.67) \mathrm{kg}$, and the median (IQR) chronological age at which procedure was done was $15(9.25,20.75)$ days. Three patients were classified as chronic lung disease at the time of inclusion in the study. Ventilator-associated pneumonia (VAP) was diagnosed in 24 (50\%) ventilatordependent patients prior to bronchoscopy. Persistent lobar atelectasis was seen in 12 ventilator-dependent patients and bronchoscopy was done with diagnostic and therapeutic intent (removal of possible mucus plug). Respiratory distress syndrome (RDS) was the most common reason for mechanical ventilation [20], followed by post-surgery [7] and meconium aspiration syndrome [6].

Table I shows bronchoscopy findings and organism profile of bronchoalveolar lavage culture. Bronchoscopy evaluations revealed airway abnormalities in 38 (79\%) patients - more than one abnormality was found in 24 $(50 \%)$. The most common finding was airway edema seen in $13(27 \%)$ patients. Laryngomalacia/ tracheomalacia or bronchomalacia was seen in $25(52 \%)$ of patients. Bronchoalveolar lavage was done in 39 patients, with adequate BAL sample collected in all. BAL culture was positive in 29 (74\%) patients; the most common organism isolated was Acinetobacter baumannii.

Overall treatment was modified in $35(73 \%)$ patients based on bronchoscopy findings/BAL culture, including tracheostomy in five patients (3, subglottic stenosis; 1 , subglottic hemangioma; 1 , severe tracheomalacia). Laser excision of subglottic stenosis through rigid bronchoscopy was done in two patients, successful

Table I Bronchoscopy and Bronchoalveolar Lavage Findings in Ventilator-Dependent Neonates $(N=48)$

\begin{tabular}{lr}
\hline Findings* & No. $(\%)$ \\
\hline Airway edema & $13(27)$ \\
Tracheomalacia & $10(20.8)$ \\
Laryngomalacia & $8(16.6)$ \\
Mucus plug & $8(16.6)$ \\
Bronchomalacia & $7(14.5)$ \\
Subglottic stenosis & $5(10.4)$ \\
BALfluid culture & \\
A. baumannii & $11(22.9)$ \\
K. pneumoniae & $10(20.8)$ \\
P. aeruginosa & $4(8.3)$ \\
S. aureus & $2(4.1)$ \\
E. coli & $1(2.0)$ \\
C. albicans & $1(2.0)$ \\
\hline
\end{tabular}

*Subglottic hemangioma, H-type fistula, right bronchial agenesis, vascular ring, and choanal stenosis in one neonate each; BAL bronchoalveolar fluid. 


\section{WHAT THIS STUDY ADDS?}

- Flexible bronchoscopy is a useful intervention in select neonates with extubation failure.

mucus plug removal for atelectasis in five patients with mucus plug (post-bronchoscopy $X$-ray $(n=3)$ showed persistent collapse of affected lobe), placement of oral airway for choanal stenosis in one patient, surgical procedure for $\mathrm{H}$ type fistula in one patient, and modification of antibiotics based on BAL culture in 21 patients. Overall 31 (64\%) patients were successfully extubated within a week of the bronchoscopy procedure, and $39(81.5 \%)$ patients could be extubated within 14 days of the procedure.

Procedural complications like transient hypoxia $(n=4)$, bradycardia $(n=2)$, transient apnea $(n=1)$ and epistaxis $(n=1)$ were seen in $8(16.7 \%)$ patients.

\section{DISCUSSION}

We found flexible bronchoscopy to be a useful diagnostic and therapeutic tool in babies on prolonged mechanical ventilation. Bronchoscopy evaluations revealed airway abnormalities in a significant number of our patients. More than half of the subjects (25/48) had laryngomalacia, tracheomalacia, or bronchomalacia, which was likely due to bronchopulmonary dysplasia and/or chronic mechanical ventilation, which are known to cause tracheobronchomalacia [9]. Flexible broncho-scopy helped us to modify treatment in $73 \%$ of ventilatordependent neonates based on the bronchoscopic/BAL culture findings.

A 7-year retrospective study on 599 neonates who underwent flexible bronchoscopy reported its importance as a diagnostic and therapeutic tool in the management of neonatal lung disease, Vijayasekaran, et al. [10] reported neonatal bronchoscopy safe in experienced hands and invaluable tools in the management of a neonate with various respiratory disorders. Others have also provided similar conclusions [6]. The most important factor responsible for ventilator dependence is ventilator-associated pneumonia [11]. Chest $X$-ray has poor sensitivity to diagnose VAP because the presence of pulmonary infiltrates on chest $X$-ray is one of the main criteria for diagnosing VAP, which may also be caused by other conditions like pulmonary edema, atelectasis or pulmonary hemorrhage [11]. Similarly, culture of the tracheal aspirate has a high chance of contamination with colonizing microorganisms [12]. BAL microbiology is a very good marker for the diagnosis of lung infection [13]. In a study by Wang, et al. [14] on risk factors of extubation failure in ELBW infants, atelectasis was also found as one of the causes of extubation failure. Extubation failure due to airway complications involving glottic, subglottic, or tracheobronchial pathology is well reported in the literature [15].

The study has some limitations. This is a review of records with no control group, and no standardization regarding the definition of prolonged mechanical ventilation; the decision for bronchoscopy was based on the clinical experience of the attending neonatologist. Secondly, the sample size is small and this was a singlecenter study.

To conclude, flexible bronchoscopy can be incorporated as a diagnostic and therapeutic modality in newborn babies with extubation failure, and we can get useful information about the cause of extubation failure.

Ethics clearance: Departmental Review Board, SIMS; No. SIMS/ 152/12/279; dated June 2, 2016.

Contributors: JIB, BAC: conceived the idea of the study and writing the manuscript; SZ: was involved in management and data collection; QIA: supervised implementation of the study; AAA: contributed to writing of the manuscript. All authors approved the final version of manuscript, and are accountable for all aspects related to the study.

Funding: None; Competing interest: None stated.

\section{REFERENCES}

1. Sant'Anna GM, Keszler M. Weaning infants from mechanical ventilation. Clin Perinatol. 2012;39:543-62.

2. Ehrenkranz RA, Walsh MC, Vohr BR, Jobe AH, Wright LL, Fanaroff AA, et al. Validation of the national institutes of health consensus definition of bronchopulmonary dysplasia. Pediatrics. 2005; 116:1353-60.

3. Walsh MC, Morris BH, Wrage LA, Vohr BR, Poole WK, Tyson JE, et al. Extremely low birthweight neonates with protracted ventilation: Mortality and 18-month neurodevelopmental outcomes. J Pediatr. 2005;146:798-804.

4. Currie A, Patel DS, Rafferty GF, Greenough A. Prediction of extubation outcome in infants using the tension time index. Arch Dis Child Fetal Neonatal Ed. 2011;96:F265-9.

5. Kurachek SC, Newth CJ, Quasney MW, Rice T, Sachdeva $\mathrm{RC}$, Patel NR, et al. Extubation failure in pediatric intensive care: A multiple-center study of risk factors and outcomes. Crit Care Med. 2003;31:2657-64.

6. Hysinger E, Friedman N, Jensen E, Zhang H, Piccione J. Bronchoscopy in neonates with severe bronchopulmonary dysplasia in the NICU. J Perinatol. 2019;39:263-8.

7. Bar-Zohar D, Sivan Y. The yield of flexible fiberoptic 
bronchoscopy in pediatric intensive care patients. Chest. 2004;126:1353-9

8. Lin YT, Lee YS, Jeng MJ, Chen WY, Tsao PC, Chan IC, et al. Flexible bronchoscopic findings and the relationship to repeated extubation failure in critical children. J Chin Med Assoc. 2018;81:804-10.

9. Downing GJ, Kilbride HW. Evaluation of airway complications in high-risk preterm infants: Application of flexible fiberoptic airway endoscopy. Pediatrics. 1995;95: 567-72.

10. Vijayasekaran D, Kalpana S, Ramachandran P, Nedunchelian K. Indications and outcome of flexible bronchoscopy in neonates. Indian J Pediatr. 2012;79:1181-4.

11. Saydain G. Ventilator-associated pneumonia in advanced lung disease: A wakeup call. Lung India. 2014;31:1-3.

12. De Blic J, Midulla F, Barbato A, Clement A, Dab I, Eber E, et al. Bronchoalveolar lavage in children. ERS task force on bronchoalveolar lavage in children. European Respiratory Society. Eur Respir J. 2000;15:217-31.

13. Bhat JI, Wani WA, Ahmad QI, Charoo BA, Ali SW, Ahangar AA, et al. Flexible bronchoscopy in non-resolving pneumonia. Indian J Pediatr. 2017;84:681-4.

14. Wang SH, Liou JY, Chen CY, Chou HC, Hsieh WS, Tsao PN. Risk factors for extubation failure in extremely low birth weight infants. Pediatr Neonatol. 2017;58:145-50.

15. Walner DL, Loewen MS, Kimura RE. Neonatal subglottic stenosis-incidence and trends. Laryngoscope. 2001;111: 48-51. 